\title{
ASO Author Reflections: Minimally Invasive Esophagectomy for Esophageal Cancer in the Prone and Lateral Position
}

\author{
Susumu Miura, $\mathrm{MD}^{1}$ (D), and Yoshihiro Kakeji, PhD, $\mathrm{MD}^{1}$ \\ Division of Gastrointestinal Surgery, Department of Surgery, Graduate School of Medicine, Kobe University, Kobe, \\ Hyogo, Japan
}

\section{PAST}

The conventional Mckeown esophagectomy for esophageal cancer has been performed with right thoracotomy. ${ }^{1}$ However, minimally invasive esophagectomy (MIE) for esophageal cancer has become a popular surgical treatment approach as well. Patients can be placed in several positions during MIE. Currently, thoracoscopic esophagectomy in the left lateral decubitus position (TEL) and thoracoscopic esophagectomy in the prone position (TEP) are frequently used. ${ }^{2,3}$

\section{PRESENT}

Several studies have suggested that TEP might be a more practical and promising procedure than TEL, with less blood loss, fewer pulmonary complications, and increased lymph node harvest. ${ }^{4}$ However, few reports compared long-term survival of TEL versus TEP. ${ }^{5}$ In our study, TEP extended operation time, but reduced blood loss, reduced pulmonary complication, and increased lymph nodes harvest compared with TEL. ${ }^{6}$ TEP also provided equal oncological efficiency compared with TEL.

ASO Author Reflections is a brief invited commentary on the article, "Long-term outcomes of thoracoscopic esophagectomy in the prone versus lateral position: a propensity score-matched analysis." Ann Surg Oncol. 2019;26:3736-44.

(C) Society of Surgical Oncology 2019

First Received: 11 October 2019;

Published Online: 28 October 2019

S. Miura, MD

e-mail: smiura@med.kobe-u.ac.jp

\section{FUTURE}

We may need to choose TEP in MIE for esophageal cancer. This is supported by the results of our study, which demonstrated that TEP provides equal oncological efficiency and reducing pulmonary complications compared with TEL. ${ }^{6}$ An alternate consideration is robotic-assisted minimally invasive esophagectomy (RAMIE), which is performed in the prone or semi-prone position. In the largescale studies and meta-analysis, including TEL and TEP, these procedures are considered equal when long-term survival is analyzed. However, TEL and TEP need to be distinguished when short-term surgical outcomes, including pulmonary complications are analyzed. ${ }^{4}$

DISCLOSURES The authors report no conflicts of interest.

\section{REFERENCES}

1. McKeown KC. Total three-stage oesophagectomy for cancer of the oesophagus. Br J Surg. 1976;63:259-62.

2. Cuschieri A, Shimi S, Banting S. Endoscopic oesophagectomy through a right thoracoscopic approach. J R Coll Surg Edinb. 1992; 37:7-11.

3. Cuschieri A. Thoracoscopic subtotal oesophagectomy. Endosc Surg Allied Technol. 1994;2:21-5.

4. Markar SR, Wiggins T, Antonowicz S, Zacharakis E, Hanna GB. Minimally invasive esophagectomy: lateral decubitus vs. prone positioning; systematic review and pooled analysis. Surg Oncol. 2015;24:212-9.

5. Kuwabara S, Kobayashi K, Kubota A, Shioi I, Yamaguchi K, Katayanagi N. Comparison of perioperative and oncological outcome of thoracoscopic esophagectomy in left decubitus position and in prone position for esophageal cancer. Langenbecks Arch Surg. 2018;403:607-14.

6. Miura S, Nakamura T, Miura Y, et al. Long-term outcomes of thoracoscopic esophagectomy in the prone versus lateral position: a propensity score-matched analysis. Ann Surg Oncol. 2019;26:3736-44.

Publisher's Note Springer Nature remains neutral with regard to jurisdictional claims in published maps and institutional affiliations. 